\title{
Amivantamab: A Potent Novel EGFR/c-MET Bispecific Antibody Therapy for EGFR-mutated Non-small Cell Lung Cancer
}

\author{
Matthew Z Guo, ${ }^{1}$ Kristen A Marrone, ${ }^{1}$ Alexander Spira, ${ }^{1,2}$ Kristine Freeman ${ }^{1,2}$ and Susan C Scott ${ }^{1}$ \\ 1. Johns Hopkins School of Medicine, Sidney Kimmel Comprehensive Cancer Center, Baltimore, MD, USA; 2. Virginia Cancer Specialists Research Institute, Fairfax, VA, USA \\ US Oncology Research, The Woodlands TX, USA
}

DOI: https://doi.org/10.17925/OHR.2021.17.1.42

W hile the majority of epidermal growth factor receptor (EGFR) driver mutations in non-small cell lung cancer (NSCLC) are sensitive to treatment with targeted tyrosine kinase inhibitors (TKIs), anti-EGFR TKIs are susceptible to resistance mechanisms through secondary mutations and also lack efficacy against tumours with non-classical EGFR mutations like exon 20 insertions. EGFR exon 20 insertions represent a challenging molecular subtype of NSCLC with no approved targeted therapy options and poor overall prognosis. Similarly, for NSCLC with EGFR mutations that are sensitive to TKIS, there are no approved targeted therapies following progression on a third-generation TKI without an alternative actionable mutation. Amivantamab is a novel, fully human bispecific anti-EGFR/c-MET antibody with clinical efficacy and favourable toxicity against both pre-treated EGFR exon 20 insertion NSCLC and classical EGFR mutations following development of TKI resistance. Preliminary findings from the phase I CHRYSALIS study report a $40 \%$ objective response rate with a median duration of response of 11.1 months for patients with pre-treated EGFR exon 20 insertion mutated tumours after platinumbased chemotherapy. Based on these results, the US Food and Drug Administration granted amivantamab accelerated approval in 2021 for treatment of adult patients with locally advanced or metastatic NSCLC with EGFR exon 20 insertion mutations whose disease has progressed on or after platinum-based chemotherapy. This review outlines the rational design and novel mechanism of action of the dual-targeted antibody, and describes preclinical and clinical data characterizing amivantamab in the treatment of NSCLC. Furthermore, we outline the practical clinical administration of this novel agent, including dosing and management of toxicities, and compare its mechanism, efficacy and safety profile to those of other EGFR exon 20 insertion targeted therapies currently available and under investigation.

\section{Keywords}

Amivantamab, EGFR, exon 20 insertion, MET, bispecific antibody, targeted therapy, NSCLC

Disclosure: Kristen A Marrone has received consulting fees from AstraZeneca and Amgen. Alexander Spira has received grant support for consulted research (institution) from Janssen and Takeda, and grant support for contracted research from Cullinan Oncology and Black Diamond. Kristine Freeman has received grant support for consulted research (institution) from Janssen. Matthew Z Guo and Susan C Scott have no financial or non-financial relationships or activities to declare in relation to this article.

Review process: Double-blind peer review.

Compliance with ethics: This study involves a review of the literature and did not involve any studies with human or animal subjects performed by any of the authors.

Authorship: The named authors meet the International Committee of Medical Journal Editors (ICMJE) criteria for authorship of this manuscript, take responsibility for the integrity of the work as a whole, and have given final approval for the version to be published.

Access: This article is freely accessible at

touchONCOLOGY.com. (c) Touch Medical Media 2021.

Received: 13 May 2021

Accepted: 21 June 2021

Published online: 21 July 2021

Citation: touchREVIEWS in Oncology \& Haematology. 2021;17(1):42-7

Corresponding author: Susan C Scott, Johns Hopkins University School of Medicine, Sidney Kimmel Comprehensive Cancer Center, 201 N Broadway, Baltimore, MD 21287, uSA. E: sscott89@jhmi.edu

Support: No funding was received in

the publication of this article.
Cancer treatment has expanded rapidly in recent years as advancements in the fields of tumour biology and molecular diagnostics have informed the development of targeted therapies, improving survival in patients with oncogene-addicted cancers with therapeutically relevant molecular lesions. Osimertinib has become the standard-of-care treatment in the USA and elsewhere for classical epidermal growth factor receptor (EGFR) driver mutations such as exon 19 deletions and exon 21 L858R substitutions that are sensitive to tyrosine kinase inhibitors (TKIs), in both the metastatic and the adjuvant settings. Nevertheless, the median duration of response (DOR) in the first line is about 17 months, ${ }^{1}$ and there are many additional EGFR mutations that do not respond to standard TKIs due to an inability of the molecule to bind the mutant receptor; the most common is the EGFR exon 20 insertion (ex20ins).

Amivantamab is a novel anti-EGFR/C-MET bispecific antibody that received US Food and Drug Administration (FDA) Breakthrough Therapy designation in March 2020 and subsequently accelerated approval in May 2021 following promising early data from the CHRYSALIS phase I study, which demonstrated both safety in advanced pre-treated non-small cell lung cancer (NSCLC) as well as clinical activity in NSCLC with EGFR ex20ins and classical EGFR mutations resistant to third-generation TKIs. ${ }^{2-4}$

This review outlines the antibody design, mechanism of action, pharmacological profile, relevant preclinical and clinical studies, and safety and tolerability of amivantamab, a promising therapeutic for non-classical EGFR ex20ins mutated and TKI-resistant NSCLC.

\section{Targeting EGFR driver mutations in non-small cell lung cancer and clinical needs}

EGFR, a transmembrane receptor tyrosine kinase in the ErbB family, mediates multiple downstream signal transduction pathways via ERK/MAPK3, PI3K/AKT and STAT that influence proliferation, apoptosis and cellular migration. 5.6 EGFR mutations are common drivers of NSCLC, with the frequency of EGFR mutations varying by ethnicity, representing approximately $15 \%$ in Caucasian populations and $50 \%$ in Asian populations, ${ }^{7}$ particularly in light/never smokers. 
Figure 1: Amivantamab mechanisms of action

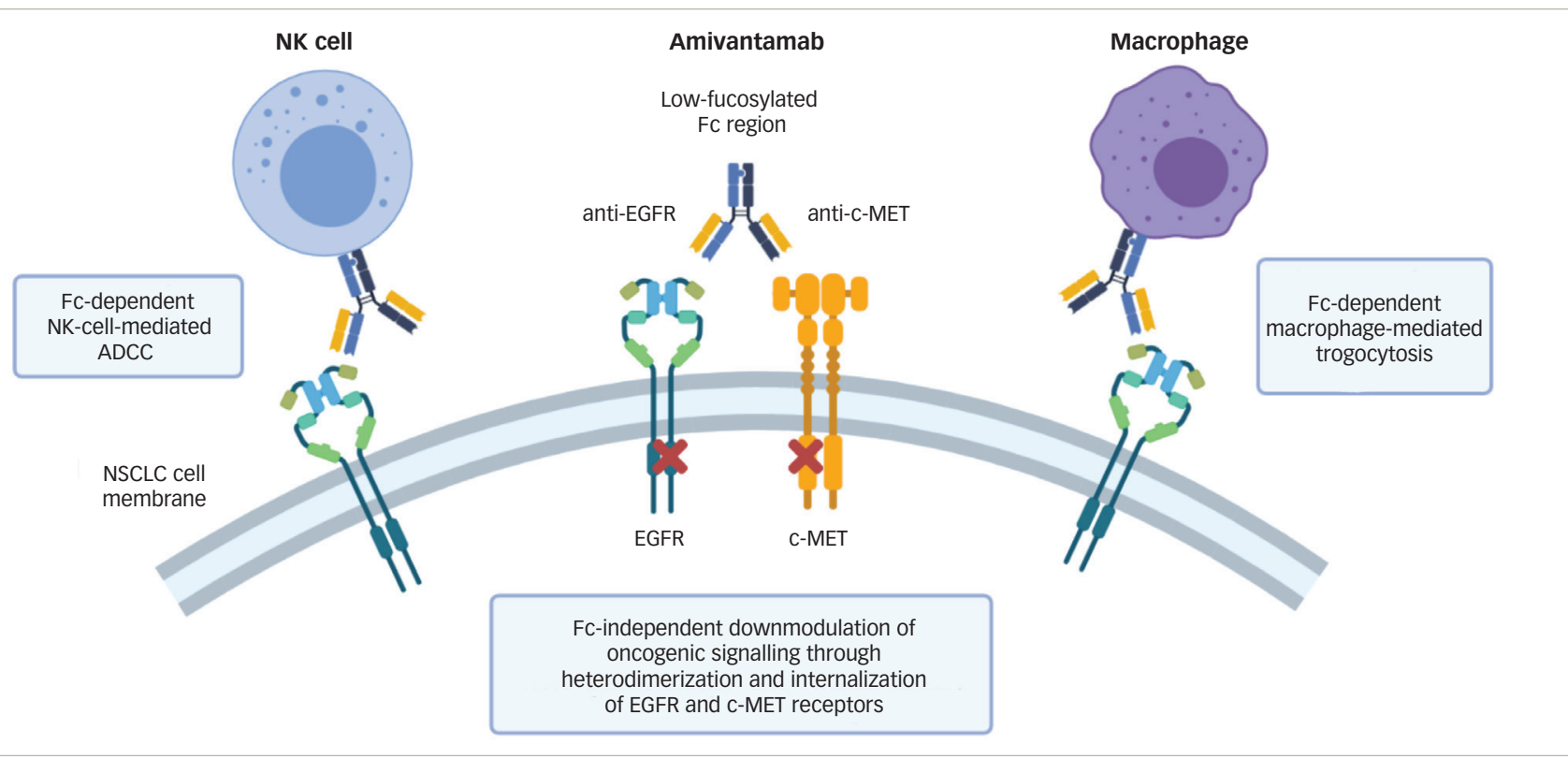

Amivantamab targets EGFR-mutated NSCLC tumour cells through FC-dependent immune cell-mediated ADCC and trogocytosis, as well as FC-independent dual EGFR/C-MET signalling downmodulation.

Figure created by author Matthew Z Guo using BioRender.com subscription.

$A D C C=$ antibody-dependent cellular cytotoxicity; EGFR = epidermal growth factor receptor; NK = natural killer; NSCLC = non-small cell lung cancer.

Clinically relevant activating mutations in the EGFR gene occur in exons 18-21 encoding the tyrosine kinase domain, and this heterogenous group of alterations, including insertions, deletions and point mutations, has variable prevalence, prognosis and response to targeted therapies. Classical EGFR primary driver mutations, such as exon 19 deletions and exon 21 L858R point mutations, account for approximately 85\% of EGFR mutations in NSCLC and are exquisitely sensitive to antiEGFR TKIs. However, resistance to first- and second-generation EGFR TKIs (gefitinib, erlotinib, afatinib and dacomitinib) develops inevitably through secondary EGFR mutations, such as exon 20 T790M mutations or upregulation of other tumour growth and metastasis-promoting pathways, such as C-MET.,9 The third-generation EGFR TKI osimertinib was developed to target tumours that acquired a T790M resistance mutation. ' With outstanding responses, osimertinib is currently approved as both a first-line therapy and as a salvage therapy for patients who progress on first- and second-generation TKI treatment. Yet, despite this success, resistance to osimertinib still develops through other on-target resistance mutations, such as EGFR C797S or other EGFR mutations or through MET amplification as a bypass pathway, as well as other nonEGFR/MET pathways or by unknown or unidentified mechanisms, ${ }^{10}$ with a median DOR of about 12-17 months. ${ }^{1,11} \mathrm{~A}$ clinical need for advancements in the treatment of classical EGFR-mutated NSCLC remains.

Insertions in exon 20 of the EGFR gene are the third most common subgroup of EGFR mutations, representing 9-12\% of EGFR mutations in NSCLC and 2-3\% of all lung adenocarcinomas. ${ }^{12,13} A$ heterogenous group of mutations, EGFR ex20ins are typically frame insertions or duplications between amino acids 762 and 774 of EGFR, encoding the C-helix and subsequent loop within the tyrosine kinase domain. ${ }^{5,12,14}$ Unlike classical EGFR mutations, ex20ins do not alter the adenosine $5^{\prime}$-triphosphate (ATP)-binding capacity of EGFR nor do they enhance affinity for ATPcompetitive TKIs. ${ }^{13,15}$ Rather, C-helix reorientation causes steric hindrance that renders current TKI therapies ineffective, as evidenced by response rates to erlotinib, gefitinib, afatinib and osimertinib of 0-8\%, ${ }^{16-20}$ leaving chemotherapy as the standard first-line approach. ${ }^{21-23}$ As these are oncogene-addicted tumours, therapies that successfully target the ex20ins would be expected to improve outcomes. Currently, the only FDA-approved targeted treatment for tumours with EGFR ex20ins mutations is amivantamab following progression on chemotherapy.

Identification of EGFR mutations by next-generation sequencing of tumour tissue or circulating tumour DNA (ctDNA) in plasma is used to determine eligibility for and choice of anti-EGFR targeted therapy. Along with the accelerated approval of amivantamab for EGFR ex20ins, the FDA approved Guardant360 ${ }^{\circledR}$ CDx (Guardant Health, Inc., Redwood City, CA, USA) as a companion diagnostic. Guardant360 CDx is a liquid biopsy test using next-generation sequencing of ctDNA to detect alterations in common driver genes, including EGFR ex20ins. Among patients in the CHRYSALIS trial, EGFR ex20ins was initially identified by next-generation sequencing of tumour tissue in $94 \%$ and by plasma in $6 \%$. Retrospectively, central testing using the Guardant360 CDx tool identified an EGFR ex20ins in $79 \%$ of available patient plasma specimens. ${ }^{24,25}$

\section{Amivantamab as a novel EGFR and MET inhibitor}

Amivantamab (JNJ-61186372) is a fully human bispecific immunoglobulin G1 (IgG1) antibody targeting both EGFR and C-MET. ${ }^{2}$ The C-MET oncoprotein is overexpressed in many NSCLC tumours at baseline, including EGFR-mutated tumours, and C-MET amplification occurs in $5-22 \%$ of tumours with acquired resistance following EGFR TKI treatment. ${ }^{26-29}$ Amivantamab was designed to both delay resistance in TKI-naïve tumours and combat resistance in TKI-resistant tumours. Initial in vitro studies found that amivantamab was able to bind to both EGFR and C-MET simultaneously with high affinity, and sequestered the two proteins in a heterodimer, preventing homodimerization and downstream signalling. ${ }^{30,31}$ Amivantamab was also designed to have low core fucosylation, ${ }^{28}$ a modification that increases binding to $\mathrm{FC} \gamma$ receptors and enhances antitumour immune cell functions. ${ }^{32}$

Preclinical studies have highlighted the multiple mechanisms by which amivantamab induces antitumour effects (Figure 1). Amivantamab 
induced natural killer-cell-mediated, antibody-dependent cellular cytotoxicity in multiple cell lines bearing classical EGFR mutations and C-MET amplifications, and also EGFR ex20ins and T790M mutations. ${ }^{28,31,33}$ In addition, amivantamab mediated tumour-cell interaction with macrophages and monocytes, resulting in trogocytosis of EGFR and c-MET receptors from the tumour cell surfaces to the immune cell surfaces and thus downmodulation of signal transduction and downstream pathways. ${ }^{33}$ Finally, additional preclinical data suggest Fc-independent mechanisms of action are triggered by inhibition of ligand binding and internalization of EGFR and c-MET receptors, leading to decreased receptor expression and further blunting of tumourigenic growth signals.,31

Overall, preclinical data suggested that amivantamab is effective in treating tumours with classical EGFR mutations, as well as ex20ins mutations and common TKI-resistance mutations. Clinically, by binding cell surface receptors instead of their intracellular tyrosine kinase domains, amivantamab is expected to overcome resistance and function mechanistically differently from small-molecule TKIs. Nevertheless, other monoclonal anti-EGFR antibodies, such as cetuximab, have met with limited efficacy in NSCLC and have substantial toxicity. ${ }^{34}$ The dualtargeting mechanism of amivantamab is hypothesized to increase avidity to tumour cells while decreasing off-target effects, overcoming some of the limitations of monospecific anti-EGFR antibodies like cetuximab.

\section{Clinical data for amivantamab in EGFR exon 20-mutated non-small cell lung cancer}

Clinical efficacy of amivantamab in advanced NSCLC has been assessed in the ongoing multi-cohort phase I CHRYSALIS study. The trial, beginning in May 2016, enrolled 460 participants with metastatic or unresectable EGFR-mutated or wild-type NSCLC who had progressed following either EGFR TKI therapy or platinum-based chemotherapy. ${ }^{35}$

The CHRYSALIS study dose escalation (part 1) determined the amivantamab recommended phase ॥ dose of 1,050 $\mathrm{mg}$ for patients weighing $<80 \mathrm{~kg}$ and $1,400 \mathrm{mg}$ for patients $\geq 80 \mathrm{~kg}$. The dose-expansion (part 2) study includes various prespecified cohorts segregated by EGFR and C-MET mutation profile. ${ }^{35}$ Initial data for patients from the ex20ins cohort included 39 response-evaluable patients, 29 (74\%) of whom had previously received platinum-based chemotherapy, 6 (15\%) were treatment-naive and 4 (10\%) had received other therapies. The overall response rate (ORR) was $36 \%$ with median DOR of 10 months. The total clinical benefit rate was $67 \%$ with median progression-free survival (PFS) of 8.3 months. The cohort included 13 distinct ex20ins mutations, and activity was observed for all mutations. ${ }^{4}$ Such responses are considerably more favourable than current outcomes of median PFS of 4.1 months for EGFR ex20ins NSCLC treated with standard-of-care chemotherapy. ${ }^{21,22}$

Follow-up data in 2021 expanded on the post-platinum EGFR ex20ins cohort, reporting outcomes for 81 patients with ORR of $40 \%$ and a total clinical benefit rate of $74 \%$. Median DOR was 11.1 months, median PFS was 8.3 months and median overall survival was 22.8 months. ${ }^{25}$ These findings prompted amivantamab FDA-accelerated approval to address the unmet need for patients with chemotherapy-refractory NSCLC with EGFR ex20ins mutations.

\section{Amivantamab in TKI-resistant EGFR-mutated non-small cell lung cancer}

In classical EGFR exon 19-deleted and L858R-mutated tumours, thirdgeneration TKIs, like osimertinib, are standard first-line treatment, yet there are no approved targeted therapies following progression without an alternative actionable mutation. Resistance to osimertinib develops through diverse mechanisms, including increased MET expression, providing a bypass pathway for dysregulated growth and proliferation signalling. 8.9 Amivantamab is expected to have antitumour effects in these cases by dual targeting of EGFR and MET, and preliminary findings of the CHRYSALIS study showed that a small number of patients with c-MET-amplified tumours following a third-generation TKI responded to amivantamab. ${ }^{3}$

Osimertinib resistance also commonly develops through a C797S mutation that prevents osimertinib binding at the tyrosine kinase domain. ${ }^{10}$ Because the $\mathrm{C} 797$ residue is intracellular, amivantamab is hypothesized to have activity against this mutation, ${ }^{31}$ and some patients in the CHRYSALIS trial who developed C797S mutations following thirdgeneration TKI therapy responded to amivantamab. ${ }^{3}$ In addition, by binding on the cell surface, amivantamab may overcome other resistance mechanisms in addition to c-MET amplification and C797S mutation that constitute a majority of post-osimertinib progression.

The combination of amivantamab with the novel third-generation antiEGFR TKI lazertinib is also under investigation in osimertinib-resistant tumours in a dose-expansion arm of CHRYSALIS. Initial data from 45 patients revealed a $36 \%$ confirmed response rate with median DOR not reached at median follow-up of 8.2 months. Additional biomarker analysis revealed increased rates of response in tumours with osimertinibresistance mutations or amplifications in EGFR/MET (response rate 47\%) compared with non-EGFR/MET mechanisms of resistance (response rate $0 \%$, and in tumours with high EGFR/MET protein expression (response rate $90 \%$ ) compared with low expression (response rate 10\%). ${ }^{36}$

\section{Clinical administration of amivantamab and management of toxicities}

Amivantamab 1,050 mg for patients $<80 \mathrm{~kg}$ and 1,400 mg for patients $\geq 80 \mathrm{~kg}$ is administered intravenously with loading doses once weekly for the first 28-day cycle and then every 2 weeks for following cycles. To monitor for and reduce infusion-related reactions, the first dose should be divided into a $350 \mathrm{mg}$ flat dose on day 1 with the remainder of the dose on day 2, while subsequent doses in cycle 1 and beyond can be given as a single full-dose infusion.

Adverse events (AES) among all patients on the CHRYSALIS study treated with amivantamab at the recommended phase $\|$ dose $(n=258)$ most commonly included rash (78\% any grade, $3 \%$ grade $\geq 3$ ), infusion-related reaction ( $65 \%$ any grade, $2 \%$ grade $\geq 3$ ) and paronychia $(40 \%)$, followed by stomatitis (19\%), pruritis (19\%) and diarrhoea (11\%). Thirty-nine percent of patients had grade $\geq 3 \mathrm{AES}$, and $16 \%$ were considered treatment-related grade $\geq 3 \mathrm{AEs}$. Treatment-related AEs uncommonly led to dose reduction (10\%) and discontinuation (3\%). There were no reports of treatmentrelated death..$^{25}$

Infusion-related reactions should trigger immediate infusion cessation and patient evaluation with acute management of respiratory symptoms, hypotension or anaphylaxis. In stable patients following symptom resolution, the infusion can be restarted at $50 \%$ reduced rate and titrated to tolerance. In study populations, nearly all infusion reactions occurred with the first infusion and rarely impacted subsequent treatments, similarly to exposure to other monoclonal antibodies like daratumumab. ${ }^{25,37}$ Patients can be premedicated with diphenhydramine and steroids, with these drugs possibly tapered after demonstrating tolerance with the initial dosing.

The side effects related to wild-type EGFR binding are very similar to those of cetuximab, particularly acne-like rash and nail changes. ${ }^{25}$ Patients should be counselled on prophylactic skin measures, including 
Table 1: Preliminary efficacy of current investigational therapies for EGFR ex20ins non-small cell lung cancer

\begin{tabular}{|c|c|c|c|c|c|}
\hline Drug & Mechanism of action & CNS penetration & Overall response rate & Median duration of response & Grade $\geq 3$ adverse events \\
\hline Amivantamab ${ }^{25}$ & Monoclonal antibody & No & $40 \%$ & 11.1 months & $16 \%$ \\
\hline Mobocertinib ${ }^{42}$ & TKI & No & $26 \%$ & 17.5 months & $46 \%$ \\
\hline Osimertinib $160 \mathrm{mg}^{48}$ & TKI & Possibly & $25 \%$ & 5.7 months & $29 \%$ \\
\hline Poziotinib ${ }^{55}$ & TKI & No & $15 \%$ & 7.4 months & $63 \%$ \\
\hline
\end{tabular}

CNS = central nervous system; EGFR = epidermal growth factor receptor; ex20ins = exon 20 insertion; TKI = tyrosine kinase inhibitor.

barrier protection with alcohol-free moisturizing creams or ointments and sunlight avoidance strategies with protective clothing and broadspectrum sunscreen. Reactive treatment measures for grade 1-2 rash include topical corticosteroids, systemic antibiotics (e.g. doxycycline, minocycline or cephalexin) to prevent bacterial superinfection, and continuation of topical moisturizers. Scalp rash may benefit from antiinflammatory, antibacterial or antifungal shampoo. In the case of grade $\geq 3$ rash, the addition of a short course of systemic steroids (e.g. prednisone $0.5 \mathrm{mg} / \mathrm{kg}$ for 7 days) can help resolve rash to grade $\leq 2$. Providers should consider dermatology consultation in patients who develop rash or other skin toxicities of grade $\geq 3$ or that do not improve within 2 weeks. For high-grade rash, treatment with low-dose acitretin or isotretinoin may be considered. Depending on the grade, treatment may be held. ${ }^{24}$

Pruritis is best managed with topical agents, such as steroids, calcineurin inhibitors or antipruritic agents. Higher-grade pruritis may benefit from oral antipruritic agents, oral corticosteroids, as well as gabapentin or pregabalin. Prophylaxis for paronychia includes measures such as skin irritant avoidance, nail moisturization and nail protection. Low-grade paronychia can improve with antimicrobial soaks and topical antiseptics with topical steroids or calcineurin inhibitors. High-grade paronychia may require the application of topical or oral antibacterial/antifungal agents. Dermatology and podiatry consults should be considered in severe or refractory cases. ${ }^{24}$ Oedema and hypoalbuminemia are common side effects as well, as seen with other c-MET inhibitors. ${ }^{25,38}$ This is generally mild and can be managed with compression hose and gentle diuresis with furosemide and spironolactone.

\section{Comparisons to other current and investigational treatments for EGFR ex20ins non-small cell lung cancer}

In addition to the bispecific antibody amivantamab, a selection of smallmolecule TKIs are under investigation in EGFR ex20ins-mutated NSCLC (Table 1). Mobocertinib is a novel TKI designed to target EGFR ex20ins that has demonstrated promising activity in preclinical studies and is currently in clinical trials. ${ }^{39}$ Preliminary results from a phase $1 /$ II study of mobocertinib in treating EGFR ex20ins NSCLC found an ORR of $56 \%$ in patients without brain metastasis at baseline, but a decreased ORR of $25 \%$ in patients with brain metastasis. ${ }^{40,41}$ In the most recently published cohort of 114 platinum pre-treated patients with EGFR ex20ins tumours, confirmed ORR was $26 \%$ with median DOR of 17.5 months and median PFS of 7.3 months. ${ }^{42}$ Similarly to other anti-EGFR therapies, mobocertinib $160 \mathrm{mg}$ had significant toxicities and was associated with diarrhoea in $90 \%$ of patients (including grade $\geq 3$ diarrhoea in $22 \%$ ), rash in $45 \%$ of patients, and required treatment discontinuation in $17 \%$ of patients. ${ }^{42}$ Neither amivantamab nor mobocertinib appear to have significant central nervous system penetration, which are limitations compared with osimertinib for common EGFR mutations. ${ }^{43}$

Osimertinib has been investigated in EGFR ex20ins tumours based on the excellent activity against classical TKI-sensitive EGFR mutations as well as preclinical data that it can bind ex20ins-mutated EGFR despite the presence of the rigid C-helix that sterically hinders the binding of first- and second-generation EGFR TKIs. ${ }^{4,45}$ The standard dose of osimertinib, $80 \mathrm{mg}$ in EGFR ex20ins NSCLC, was investigated in two small single-arm clinical trials, both reporting no objective responses (ORR 0\%). ${ }^{19,46}$ In tumours with classical EGFR mutations and leptomeningeal disease, the BLOOM study demonstrated that a higher dose of osimertinib $160 \mathrm{mg}$ provided clinically meaningful benefit and was generally well tolerated. ${ }^{47}$ Following this, a phase II study of patients with EGFR ex20ins NSCLC treated with osimertinib $160 \mathrm{mg}$ reported a confirmed ORR of 25\% (5 of 20 patients including one complete response), and an impressive disease control rate of $85 \%$ with a median PFS of 9.7 months. ${ }^{48}$ The increased dose was well tolerated, and reported toxicities were similar to those expected with osimertinib, most prominently diarrhoea, followed by fatigue, thrombocytopaenia and rash. ${ }^{48}$ These results merit further study of whether dose escalation for osimertinib can improve outcomes in patients with EGFR ex20ins in NSCLC.

Poziotinib is a novel EGFR inhibitor that was designed as a smaller molecule than current first-generation EGFR TKIs, such as afatinib, allowing it to bypass the steric hindrance induced by ex20ins mutations. ${ }^{49}$ An initial phase II study of poziotinib in EGFR ex20ins NSCLC yielded a $44 \%$ ORR and a median PFS of 5.5 months. ${ }^{50}$ However, the follow-up multicentre ZENITH2O study demonstrated a lower ORR of 15\% with poziotinib treatment for pre-treated ex20ins-mutated tumours with a median PFS of 4.2 months among 115 patients..$^{51}$ Poziotinib also has considerable off-target EGFR wild-type activity, contributing to the high rate of treatment-related AEs identified in both clinical trials, including rash and diarrhoea, with frequent dose reductions or discontinuation. .0.51 $^{5}$ Alternative dosing regimens are being studied to overcome the substantial toxicity. ${ }^{52}$ Other drugs targeting EGFR ex20ins are currently in development as well (including CLN-081 and BDTX-189). ${ }^{53,54}$

\section{Limitations and resistance to amivantamab}

Amivantamab was designed to overcome resistance mechanisms to anti-EGFR TKIs through its bispecific antibody construct with extracellular binding of EGFR and dual engagement of MET. Though the clinical efficacy in both EGFR ex20ins and TKI resistance mutations is encouraging, only a subset of patients responds to amivantamab. Importantly, as a monoclonal antibody, amivantamab is expected to have poor bloodbrain barrier penetration, ${ }^{55}$ and so its use may be limited in patients with intracranial metastases. Additionally, many patients have developed progressive disease on amivantamab therapy, ${ }^{3,4}$ highlighting the need to further explore the underpinnings of sensitivity and acquired resistance. Future studies and analyses will help determine the relative sensitivities of different exon 20 mutations to amivantamab and identify potential biomarkers predictive of response.

\section{Current and future trials of amivantamab in NSCLC}

Several trials of amivantamab are ongoing to investigate its potential for the treatment of NSCLC (Table 2). Part 2 of the CHRYSALIS study continues 
Table 2: Ongoing trials for amivantamab in non-small cell lung cancer

\begin{tabular}{|c|c|c|c|c|}
\hline Trial & Phase & Study treatment & Patient population & Current status \\
\hline CHRYSALIS Part $2^{35}$ & $\mid \mathrm{b} / \mathrm{I}$ & $\begin{array}{l}\text { Amivantamab or amivantamab plus } \\
\text { lazertinib }\end{array}$ & $\begin{array}{l}\text { Pre-treated NSCLC with EGFR ex20ins mutation, } \\
\text { classical EGFR mutation with secondary TKI } \\
\text { resistance, or METex14 skipping }\end{array}$ & $\begin{array}{l}\text { Recruiting, estimated completion } \\
\text { January } 2024\end{array}$ \\
\hline CHRYSALIS- $2^{58}$ & $\mathrm{I} / \mathrm{Ib}$ & Amivantamab plus lazertinib & $\begin{array}{l}\text { Pre-treated NSCLC with EGFR ex19del or } \\
\text { L858R mutation post-osimertinib, or pre-treated } \\
\text { NSCLC with EGFR ex20ins or other uncommon } \\
\text { EGFR mutations }\end{array}$ & $\begin{array}{l}\text { Recruiting, estimated completion } \\
\text { October } 2024\end{array}$ \\
\hline PAPILLON ${ }^{56}$ & III & $\begin{array}{l}\text { Amivantamab plus carboplatin- } \\
\text { pemetrexed versus carboplatin- } \\
\text { pemetrexed }\end{array}$ & Treatment-naïve NSCLC with EGFR ex20ins & $\begin{array}{l}\text { Recruiting, estimated completion } \\
\text { November } 2024\end{array}$ \\
\hline MARIPOSA $^{59}$ & III & $\begin{array}{l}\text { Amivantamab plus lazertinib versus } \\
\text { osimertinib }\end{array}$ & $\begin{array}{l}\text { Treatment-naïve NSCLC with EGFR ex19del or } \\
\text { L858R mutation }\end{array}$ & $\begin{array}{l}\text { Recruiting, estimated completion } \\
\text { March } 2026\end{array}$ \\
\hline
\end{tabular}

EGFR = epidermal growth factor receptor; ex19del = exon 19 deletion; ex20ins = exon 20 insertion; METex14 = MET exon 14; NSCLC = non-small cell lung cancer; TKI = tyrosine kinase inhibitor.

and will elucidate safety and preliminary efficacy for amivantamab in several cohorts, including EGFR ex20ins, classically EGFR-mutated NSCLC following progression on a third-generation EGFR TKI, classically EGFR-mutated NSCLC with documented C797S mutation or secondary MET amplification, and NSCLC with MET exon 14 skipping alterations. ${ }^{35}$

PAPILLON, a phase III study comparing first-line amivantamab plus carboplatin-pemetrexed with carboplatin-pemetrexed chemotherapy alone for advanced NSCLC, began in October 2020 and is currently recruiting patients with EGFR ex20ins mutations. The primary endpoint is PFS, and the study design includes optional cross-over to secondline amivantamab for patients in the chemotherapy only arm. ${ }^{56}$ PAPILLON thus seeks to understand if amivantamab can supplement first-line chemotherapy and extend PFS in patients with poor-risk EGFR ex20ins tumour mutations. Given the second-line response rates, it is unclear if targeted therapy alone with single-agent amivantamab could eventually replace chemotherapy for treatment-naïve patients, but subsequent studies may investigate this possibility. Other applications of amivantamab in EGFR ex20ins, such as use in the adjuvant setting, may be looked at in the future.

For patients with tumours harbouring EGFR mutations sensitive to TKIS, preclinical studies have found that lazertinib has higher selectivity, fewer off-target effects, and greater intracranial penetration than osimertinib, ${ }^{57}$ and the combination of amivantamab and lazertinib demonstrated efficacy in patients after relapse on osimertinib in a CHRYSALIS dose expansion arm. ${ }^{36}$ Next, the CHRYSALIS-2 trial seeks to study this combination in larger cohorts to prospectively validate biomarkers including response rates by different mechanisms of osimertinib resistance, and the study will also include pre-treated EGFR ex20ins and other uncommon EGFR mutation cohorts. ${ }^{58}$ Similarly, MARIPOSA, a phase III study of amivantamab and lazertinib combination therapy against osimertinib for NSCLC with EGFR exon 19 deletions or exon 21 L858R substitutions, began in September 2020 and is currently recruiting treatment-naive patients. ${ }^{59}$ MARIPOSA thus seeks to demonstrate whether amivantamab and lazertinib combination therapy or lazertinib monotherapy can function as first-line therapies comparable to osimertinib monotherapy for EGFR-mutated NSCLC.

\section{Conclusion}

With its novel mechanism of action targeting dual EGFR and c-MET extracellular domains, the bispecific fully human antibody amivantamab has activity in both NSCLC with EGFR exon 20 insertion mutations and third-generation EGFR TKI-resistant NSCLC, demonstrated in various preclinical and clinical studies described above. The toxicity profile of amivantamab is favourable due to the bispecific nature of the antibody, making it a safe and viable therapeutic option for patients with both classical and non-classical EGFR mutations who have relapsed on standard first-line therapies. Compared with novel TKIs under investigation for EGFR ex20ins, including mobocertinib and poziotinib, amivantamab has demonstrated superior or equivalent response rates and significantly less toxicity in this population. Current and future studies evaluating amivantamab usage in combination therapies or as a first-line agent will be important and exciting to follow. $\square$
1. Soria J-C, Ohe Y, Vansteenkiste J, et al. Osimertinib in untreated EGFR-mutated advanced non-small-cell lung cancer. N Eng/ J Med. 2018;378:113-25.

2. Yun J, Lee S-H, Kim S-Y, et al. Antitumor activity of amivantamab (JNJ-61186372), an EGFR-CMet bispecific antibody, in diverse models of EGFR exon 20 insertion-driven NSCLC. Cancer Discov. 2020;10:1194-209.

3. Haura EB, Cho BC, Lee JS, et al. JNJ-61186372 (JNJ-372), an EGFR-CMet bispecific antibody, in EGFR-driven advanced non-small cell lung cancer (NSCLC). J Clin Oncol. 2019;37(Suppl. non-small

4. Park K, John T, Kim S-W, et al. Amivantamab (JNJ-61186372), an anti-EGFR-MET bispecific antibody, in patients with EGFR exon 20 insertion (exon20ins)-mutated non-small cell lung cancer (NSCLC). J Clin Oncol. 2020;38(Suppl. 15):9512.

5. Yasuda H, Kobayashi S, Costa DB. EGFR exon 20 insertion mutations in non-small-cell lung cancer: preclinical data and clinical implications. Lancet Oncol. 2012;13:e23-31.

6. da Cunha Santos G, Shepherd FA, Tsao MS. EGFR mutations and lung cancer. Annu Rev Pathol. 2011;6:49-69.

7. Midha A, Dearden S, McCormack R. EGFR mutation incidence in non-small-cell lung cancer of adenocarcinoma histology: a systematic review and global map by ethnicity (mutMapII). Am . Cancer Res. 2015;5:2892-911.

8. Gazdar AF. Activating and resistance mutations of EGFR in non- small-cell lung cancer: role in clinical response to EGFR tyrosine kinase inhibitors. Oncogene. 2009;28(Suppl. 1):S24-31.

9. Turke AB, Zejnullahu K, Wu Y-L, et al. Preexistence and clona selection of MET amplification in EGFR mutant NSCLC. Cancer Cell. 2010;17:77-88.

10. Leonetti A, Sharma S, Minari R, et al. Resistance mechanisms to osimertinib in EGFR-mutated non-small cell lung cancer. $\mathrm{Br}$ Cancer. 2019;121:725-37.

11. Ahn M, Tsai C, Shepherd FA, et al. Osimertinib in patients with T790M mutation-positive, advanced non-small cell lung cance Ton-term follow-up from a pooled analysis of 2 phase 2
long long-term follow-up from a pooled
studies. Cancer. 2019;125:892-901.

Studies. Cancer. 2019;125:892-901.
12. Arcila ME, Nafa K, Chaft JE, et al. EGFR exon 20 insertion mutations in lung adenocarcinomas: prevalence, molecular heterogeneity, and clinicopathologic characteristics. Mol Cancer Ther. 2013;12:220-9.

13. Riess JW, Gandara DR, Frampton GM, et al. Diverse EGFR exon 20 insertions and co-occurring molecular alterations identified by comprehensive genomic profiling of NSCLC. J Thorac Oncol. 2018;13:1560-8.

14. Remon J, Hendriks LEL, Cardona AF, Besse B. EGFR exon 20 insertions in advanced non-small cell lung cancer. a new history begins. Cancer Treat Rev. 2020;90:102105.

15. Yasuda H, Park E, Yun C-H, et al. Structural, biochemical, and clinical characterization of epidermal growth factor receptor
(EGFR) exon 20 insertion mutations in lung cancer. Sci Trans/ Med. 2013;5:216ra177.

16. Kate S, Chougule A, Joshi A, et al. Outcome of uncommon EGFR mutation positive newly diagnosed advanced non-small cell lung cancer patients: a single center retrospective analysis. Lung Cancer (AuCKI). 2019;10:1-10.

17. Cheng G, Song Z, Chen D. Clinical efficacy of first-generation EGFR-TKIs in patients with advanced non-small-cell lung cancer harboring EGFR exon 20 mutations. Onco Targets Ther. cancer harboring EGFR exon 20 mutations. Onco Targets Ther.

18. Yang JC-H, Sequist LV, Geater SL, et al. Clinical activity of afatinib in patients with advanced non-small-cell lung cancer harbouring uncommon EGFR mutations: a combined post-hoc analysis of LUX-Lung 2, LUX-Lung 3, and LUX-Lung 6. Lancet Oncol. 2015;16:830-8.

19. Kim TM, Ock C-Y, Kim M, et al. Phase II study of osimertinib in NSCLC patients with EGFR exon 20 insertion mutation: a multicenter trial of the Korean Cancer Study Group (LU17-19). Ann Oncol. 2019;30:v628.

20. van Veggel B, Madeira R Santos JFV, Hashemi SMS, et al. Osimertinib treatment for patients with EGFR exon 20 mutation positive non-small cell lung cancer. Lung Cancer. 2020;141:9-13.

21. Wang $Y$, Yang $G$, Li J, et al. Real-world treatment outcome of advanced Chinese NSCLC EGFR exon 20 insertion patients. J 
Clin Oncol. 2019;37(Suppl 15):9043.

22. Wu J-Y, YU C-J, Shih J-Y. Effectiveness of treatments for advanced non-small-cell lung cancer with exon 20 insertion epidermal growth factor receptor mutations. Clin Lung Cancer. 2019;20:e620-30.

23. DerSarkissian M, Li S, Galaznik A, et al. HSR19-084: Real-world treatment patterns and clinical outcomes in non-small cell lung cancer patients with egfr exon 20 insertion mutations. I Natl Compr Canc Netw. 2019;17:HSR19-084.

24. RYBREVANT (amivantamab-vmjw) highlights of prescribing information. 2021. Available at: www.accessdata.fda.gov/ drugsatfda_docs/label/2021/761210s000lbl.pdf (accessed 28 June 2021)

25. Sabari JK, Catherine A Shu, Keunchil Park, et al. Amivantamab in post-platinum EGFR exon 20 insertion mutant non-small cell lung cancer. Presented at the: 2020 World Conference on Lung Cancer; 29 January 2021; IASLC.

26. Matsubara D, Ishikawa S, Sachiko O, et al. Co-activation of epidermal growth factor receptor and C-MET defines a distinct epidermal growth factor receptor and C-MET defines a distinct subset of lung adenocarcinomas. Am J Pathol. 2010;177:2191-
204.

27. Wang $Q$, Yang $S$, Wang $K$, Sun S-Y. MET inhibitors for targeted therapy of EGFR TKI-resistant lung cancer. J Hematol Oncol. 2019;12:63

28. Grugan $\mathrm{KD}$, Dorn K, Jarantow SW, et al. Fc-mediated activity of EGFR x C-Met bispecific antibody JNJ-61186372 enhanced killin of lung cancer cells. MAbs. 2017;9:114-26.

29. Guo B, Cen H, Tan X, et al. Prognostic value of MET gene copy number and protein expression in patients with surgically resected non-small cell lung cancer. a meta-analysis of published literatures. PLOS ONE. 2014;9:e99399.

30. Jarantow SW, Bushey BS, Pardinas JR, et al. Impact of cellsurface antigen expression on target engagement and function of an epidermal growth factor receptor $\times$ C-MET bispecific of antibody. J Biol Chem. 2015;290:24689-704.

31. Moores SL, Chiu ML, Bushey BS, et al. A novel bispecific antibody targeting EGFR and cMet Is effective against EGFR inhibitor-resistant lung tumors Cancer Res. 2016:76:3942-53.

32. Shields RL, Lai J, Keck R, et al. Lack of fucose on human IgG1 $\mathrm{N}$-linked oligosaccharide improves binding to human Fcgamm RIII and antibody-dependent cellular toxicity. J Biol Chem. 2002;277:26733-40.

33. Vijayaraghavan $\mathrm{S}$, Lipfert $\mathrm{L}$, Chevalier K, et al. Amivantamab (JNJ-61186372), an FC enhanced EGFR/CMet bispecific antibody, induces receptor downmodulation and antitumor activity by monocyte/macrophage trogocytosis. Mol Cancer Ther: 2020;19:2044-56.

34. Hasegawa $\mathrm{H}$, Yasuda $\mathrm{H}$, Hamamoto J, et al. Efficacy of afatinib or osimertinib plus cetuximab combination therapy for nonsmall-cell lung cancer with EGFR exon 20 insertion mutations Lung Cancer. 2019:127:146-52.
35. ClinicalTrials.gov. Study of Amivantamab, a Human Bispecific EGFR and cMet Antibody, in Participants With Advanced NonSmall Cell Lung Cancer (CHRYSALS): NCT02609776. Avalable Small Cell tung Cancer (CHRYSALIS): NCTO2609776. Available at: https://clinicaltrials.gov/ct2/show/NCT02609776 (accessed
28 June 2021). 28 June 20

6. Bauml J, Cho BC, Park K, et al. Amivantamab in combination with lazertinib for the treatment of osimertinib-relapsed, chemotherapy-naïv EGFR mutant (EGFRm) non-small cell lung cancer (NSCLC) and potential biomarkers for response. J Clin Oncol. 2021;39(Suppl. 15):9006.

37. Palumbo A, Chanan-Khan A, Weisel K, et al. Daratumumab, bortezomib, and dexamethasone for multiple myeloma. N Eng J Med. 2016;375:754-66.

38. Wolf J, Seto T, Han J-Y, et al. Capmatinib in MET Exon 14-Mutated or MET-Amplified Non-Small-Cell Lung Cancer. N Eng/ J Med. 2020;383(10):944-57.

39. Gonzalvez F, Vincent S, Baker TE, et al. Mobocertinib (TAK-788): a targeted inhibitor of EGFR Exon 20 insertion mutants in nonsmall cell lung cancer. Cancer Discov. 2021; candisc.1683.2020 doi: 10.1158/2159-8290.CD-20-1683. Online ahead of print.

40. Janne PA, Neal JW, Camidge DR, et al. Antitumor activity of TAK-788 in NSCLC with EGFR exon 20 insertions. I Clin Oncol. TAK-788 in NSCLC with EGF
2019;37(Suppl. 15):9007.

41. Riely GJ, Neal JW, Camidge DR, et al. Activity and safety of mobocertinib (TAK-788) in previously treated non-small cel lung cancer with EGFR exon 20 insertion mutations from a phase I/I trial. Cancer Discov. 2021; doi: 10.1158/2159-8290 CD-20-1598. Online ahead of print.

42. Zhou C, Ramalingam S, Li B, et al. OA04.03 mobocertinib in NSCLC with EGFR exon 20 insertions: results from EXCLAIM and pooled platinum-pretreated patient populations. $J$ Thorac Oncol. 2021;16(Suppl. 3):S108.

43. Wu Y Y L, Ahn M-J, Garassino MC, et al. CNS efficacy of osimertinib in patients with $7790 \mathrm{M}$-positive advanced nonsmall-cell lung cancer. data from a randomized phase III tria Small-cell lung cancer. data from a randor
(AURA3). J Clin Oncol. 2018;36:2702-9.

44. Hirano $\mathrm{T}$, Yasuda $\mathrm{H}$, Tani $\mathrm{T}$, et al. In vitro modeling to determine Hirano T, Yasuda H, Tani T, et al. In vitro modeling to determine
mutation specificity of EGFR tyrosine kinase inhibitors against mutation specificity of EGFR tyrosine kinase inhibitors against
clinically relevant EGFR mutants in non-small-cell lung cancer. clinically relevant EGFR mutants
Oncotarget. 2015;6:38789-803.

Oncotarget. 2015;6:38789-803.
45. Floc'h N, Martin MJ, Riess JW, et al. Antitumor activity of osimertinib, an irreversible mutant-selective EGFR tyrosine kinase inhibitor, in NSCLC harboring EGFR exon 20 insertions. Mol Cancer Ther. 2018;17:885-96.

46. Ichihara E, Yasuda H, Takashima Y, et al. CT106 - Phase I/II study of osimertinib in EGFR exon 20 insertion mutations in non-small cell lung cancer patients: AEX20. Presented at the: AACR Virtua Annual Meeting 2021; 10 April 2021.

47. Yang JCH, Kim S-W, Kim D-W, et al. Osimertinib in patients with epidermal growth factor receptor mutation-positive non-smallcell lung cancer and leptomeningeal metastases: the BLOOM study. J Clin Oncol. 2020;38:538-47.

48. Piotrowska Z, Wang Y, Sequist LV, Ramalingam SS. ECOG-ACRIN 5162: a phase II study of osimertinib $160 \mathrm{mg}$ in NSCLC with
EGFR exon 20 insertions. J Clin Oncol. 2020;38(Suppl. 15):9513

49. RobichauX JP. Elamin YY, Tan Z, et al. Mechanisms and clinical activity of an EGFR and HER2 exon 20-selective kinase inhibitor in non-small cell lung cancer. Nat Med. 2018;24:638-46.

50. Heymach J, Negrao M, Robichaux J, et al. OA02.06 a phase II trial of poziotinib in EGFR and HER2 exon 20 mutant non-smal cell lung cancer (NSCLC). J Thorac Oncol. 2018;13:S323-4.

51. Le X, Goldman JW, Clarke JM, et al. Poziotinib shows activity and durability of responses in subgroups of previously treated EGFR exon 20 NSCLC patients. J Clin Oncol. 2020;38(Suppl 15):9514.

52. Le X, Shum E, Suga JM, et al. Poziotinib administered twice daily improves safety and tolerability in patients with EGFR or HER2 exon 20 mutant NSCLC (ZENITH20-5). Cancer Res. 2021;81(Suppl. 13):CT169.

53. Piotrowska Z, Nguyen D, Koczywas M, et al. 1345P Preliminary safety and activity of CLN-081 in NSCLC with EGFR exon 20 insertion mutations (Ins20). Ann Oncol. 2020:31(Suppl. 4):S862.

54. ClinicalTrials.gov. A Study of BDTX-189, an Orally Available Allosteric ErbB Inhibitor, in Patients With Advanced Solid Allosteric ErbB Inhibitor, in Patients With Advanced Sold
Tumors. (MasterKey-01). ClinicalTrials.gov Identifier: Tumors. (Masterkey-01). Cinicaltrials. gov Identifler:
NCT04209465. Available at: https://clinicaltrials.gov/ct2/show/ NCT04209465. Available at: https://Clinicat
NCT04209465 (accessed 28 June 2021).

55. Bickel U. Antibody delivery through the blood--brain barrier. Adv Drug Deliv Rev. 1995; 15:53-72.

56. ClinicalTrials.gov. A Study of Combination Amivantamab and Carboplatin-Pemetrexed Therapy, Compared With CarboplatinPemetrexed, in Participants With Advanced or Metastatic Non-Small Cell Lung Cancer Characterized by Epidermal Growth Factor Receptor (EGFR) EXon 20 Insertions (PAPILLON).
ClinicalTrials.gov Identifier: NCT04538664. Available at: https:// clinicaltrials.gov/ct2/show/NCT04538664 (accessed 28 June 2021).

57. Ahn M-J, Han J-Y, Lee KH, et al. Lazertinib in patients with EGFR mutation-positive advanced non-small-cell lung cancer: results from the dose escalation and dose expansion parts of a first-inhuman, open-label, multicentre, phase 1-2 study. Lancet Oncol. 2019;20:1681-90.

58. ClinicalTrials.gov. A Study of Lazertinib as Monotherapy or in Combination With Amivantamab in Participants With Advanced Non-small Cell Lung Cancer. ClinicalTrials.gov Identifier. NCT04077463. Available at: https://clinicaltrials.gov/ct2/show/ NCT04077463 (accessed 28 June 2021).

59. ClinicalTrials.gov. A Study of Amivantamab and Lazertinib Combination Therapy Versus Osimertinib in Locally Advanced or Metastatic Non-Small Cell Lung Cancer (MARIPOSA). Clinical Trials.gov Identifier: NCT04487080. Available at: https:// clinicaltrials. gov/ct2/show/NCT04487080 (accessed 28 June 2021). 